\title{
ReiDoCrea un proyecto de construcción y divulgación del conocimiento
}

\author{
ReiDoCrea a Project of Knowledge Building and Dissemination \\ José Miguel García Ramírez. Universidad de Granada \\ Fecha de recepción: 30 de mayo de 2013 | Fecha de aceptación y publicación: 12 de junio de 2013
}

\section{Resumen}

ReiDoCrea es un proyecto de innovación que consolida la primera revista electrónica de investigación en la que los estudiantes pueden construir y divulgar el conocimiento aprendido mientras estudian. El proyecto utiliza los principios de Investigación-Acción. Durante el curso académico 2011-12 participaron 145 estudiantes ( $M=26,52$, $S D=5,23)$. Se publicaron 21 artículos de 39 presentados. Las categorías emergentes significativas fueron: Motivación con un $92 \%$ de frecuencia y Dificultad con un $89 \%$ de frecuencia (Atla.ti).

Palabras clave: Educación | Creatividad | Innovación | Empatía | Motivación

\begin{abstract}
ReiDoCrea is an innovation project that consolidates the first electronic research journal in which students can build and disseminate knowledge learned while studying. The project uses the principles of action research. During the academic year 2011-12 145 students participated $(M=26.52, S D=5.23)$. Of the 39 articles presented, 21 were published. Significant emerging categories were: Motivation with $92 \%$ frequency and difficulty with $89 \%$ frequency (Atla.ti).
\end{abstract}

Keywords: Education | Creativity | Innovation | Empathy | Motivation

La innovación y la creatividad son claves en el desarrollo de la Educación Superior. En este sentido, el pensamiento creativo es necesario tanto en el alumnado como en el profesorado para favorecer e incrementar la eficiencia y la satisfacción en el proceso educativo. Además, la sociedad reclama una enseñanza renovada, creativa, interdisciplinar y contextualizada (Fiorini y Garcia-Ramirez, 2013; Garcia-Ramirez, Garcia-Sempere y Fiorini, 2012; Garcia-Ramirez, 2012).

A través del uso de las Tecnologías de la Información y la Comunicación, TIC, se implementa el proyecto de innovación docente ReiDoCrea, consolidándose como revista electrónica de investigación en la que los estudiantes pueden construir y divulgar el conocimiento aprendido durante el curso académico. Por lo tanto, para consolidar el proyecto se utilizan los principios de Investigación-Acción, para generar un cambio de actitud y comportamiento, promocionar el aprendizaje social y cooperativo, y potenciar el desarrollo personal de identidad sociocultural (GarciaRamirez, 2012). Esto posibilita el cumplimiento de los objetivos marcados por el proyecto: Mejorar la docencia, como proceso de enseñanza-aprendizaje; Incrementar la satisfacción y la motivación en docentes y estudiantes; Aumentar la motivación en la educación universitaria; Desarrollar un nuevo instrumento de evaluación del rendimiento académico; y divulgar el conocimiento adquirido por el alumnado. 
La Universidad de Granada, para cumplir con una educación con garantía de la calidad, evalúa anualmente la docencia a través de un cuestionario aprobado por su Consejo de Gobierno. La valoración media en Facultad de Ciencias de la Educación es de aproximadamente 4,00 sobre 5,00 . Aunque el rendimiento y la eficacia de la docencia son altos, se puede mejorar motivando a los estudiantes a publicar sus trabajos para divulgarlos en la sociedad. (García-Ramírez, 2012)

\section{Método}

\section{Participantes}

Participaron 145 estudiantes, 113 mujeres y 32 hombres, con edades comprendidas entre 20 y 61 años $(M=26,52$, $S D=5,23)$. Los participantes participaron voluntariamente y eran estudiantes de las titulaciones o grado de Psicopedagogía $(46 \%)$, Pedagogía (5\%), Primaria (5\%), Educación Social (18\%), Educación Infantil (5\%) Psicología (15\%) y Logopedia (6\%).

\section{Procedimiento e instrumentos}

Durante el curso académico 2011-12 los/as participantes tenían que elaborar un estudio de investigación relacionado con unas asignatura que estaban cursando en ese momento, siguiendo las normas publicadas en la revista ReiDoCrea. Después de finalizarlo y de que fuese evaluado por su profesorado se remitía a la revista para su segunda evaluación y publicación si procedía; luego los/as participante tenían que completar un cuestionario de una pregunta abierta en el que se le pedía que describiera su experiencia, expresando como se sintieron durante todo el proceso de elaboración, revisión y publicación de su trabajo.

Se aplicó la valoración de logro; entendiendo como logro la publicación de su trabajo académico el cual había sido evaluado por pasos de manera estrictamente sistemática:

- La primera la realizó el profesorado participante, evaluando los trabajos realizados por el alumnado. Previamente el profesorado había incorporado, en el contenido de la guía docente, los criterios de evaluación correspondientes este trabajo.

- La segunda valoración de los trabajos fue realizada por los miembros del consejo o evaluadores externos.

- La tercera valoración de los trabajos fue realizada por un comité de iguales, estudiantes, para encontrar los puntos fuerte, débiles y de mejora.

- La cuarta valoración es una autoevaluación, a través de un relato breve (máximo de 200 palabras), para explicar su experiencia en esta actividad. Los datos obtenidos se analizan a través del programa Atla.ti para codificar el material y describir las categorías emergentes.

\section{Resultados}

Los/as 145 participantes presentaron 39 artículos valorados positivamente por su profesorado. Todos los/as estudiantes participantes superaron las asignaturas, el $52 \%$ de los/as participantes obtuvieron sobresaliente y el $26 \%$ notable.

Posteriormente los trabajos fueron enviados a la revista para su segunda valoración y posible publicación en la revista. De los 39 artículos presentados fueron evaluados positivamente y publicados 21 artículos elaborados por 61 participantes, equivale al $41 \%$ de los 145 participantes. 
La tercera valoración se hizo entre iguales para conocer los puntos fuertes, débiles y posibilidades de mejora; se exponen en la siguiente tabla:

\begin{tabular}{lll} 
PUNTOS FUERTES & PUNTOS DÉBILES & POSIBILIDADES DE MEJORA \\
\hline Motivación. & & \\
\hline $\begin{array}{l}\text { Revista electrónica de investigación } \\
\text { gratuita y de carácter público. }\end{array}$ & Redactar un artículo. & $\begin{array}{l}\text { Curso específicos para } \\
\text { elaborar artículos académicos } \\
\text { Depositada en el Digibug. }\end{array}$ \\
\hline Satisfacción. & & \\
\hline $\begin{array}{l}\text { Publicación de los trabajos } \\
\text { elaborados por los estudiantes. }\end{array}$ & Tiempo de revisión. \\
\hline
\end{tabular}

La última valoración corresponde al cuestionario en el que se le pedía a la persona participante que describiera su experiencia, expresando como se sintió durante todo el proceso de elaboración, revisión y publicación de su trabajo. Los/as participantes entregaron el cuestionario completado, después de saber que artículos fueron evaluados positivamente para su publicación. Participaron el $100 \%$ de los participantes. Las categorías emergentes significativas fueron: Motivación con un $92 \%$ de frecuencia y Dificultad con un $89 \%$ de frecuencia (Atla.ti). La dificultad se debía, según los datos obtenidos, a la inseguridad y falta de apoyo en el momento de elaborar un artículo académico, principalmente, porque no tuvieron formación previa de cómo elaborar un artículo.

A continuación se detallan la información básica de los artículos aceptados: Título, autores/as, resumen y palabras clave:

DOCENCIA-CREATIVA. Una estrategia de motivación a través de la difusión del conocimiento (García-Ramírez, 2012). Resumen: Docencia-Creativa es un proyecto de innovación docente de la Universidad de Granada con la misión de publicar los trabajos que realiza el alumnado durante el curso académico. Supone una mejora global en el proceso de enseñanza-aprendizaje, porque implica la participación activa y creativa de ambas partes del proceso educativo, docentes y estudiantes. Palabras clave: Educación; Creatividad; Innovación; Empatía; Motivación.

El efecto del burnout en la antigüedad en el puesto de trabajo: Análisis mediacional de la ansiedad (Sagripanti, Gonzalez, Messi, Romero y Khlie, 2012). Resumen: Cada vez en mayor medida se hace patente que el síndrome de burnout es una realidad social. Esta disfunción psicológica está relacionada directamente con el ámbito laboral, que afecta especialmente a la profesión sanitaria, caracterizada por una continua relación de ayuda indispensable hacia otras personas, que a su vez dependen del citado personal. La muestra de estudio es de 70 trabajadores de un mismo centro del sector sanitario de atención de mayores de la ciudad de Granada. Los resultados muestran que la ansiedad se relacionará positivamente tanto con el burnout como con la antigüedad en el puesto, mientras que predecimos que a menor realización personal en presencia de mayor antigüedad los valores en ansiedad también serán más altos. También se comprueba que la ansiedad estado tiene efectos mediadores sobre la realización personal, puesto que puede causar mayor alteración de este estado que la propia antigüedad. Palabras Clave: Síndrome de estar quemado; Antigüedad; Realización personal; Sanidad; Estrés.

Cualificación y satisfacción laboral: Un estudio sobre los empleos para los que hemos sido formados previamente (Asenjo, Dios y Banqueri, (2012). Resumen: Esta investigación, que ha contado con 80 sujetos, explora la relación entre las diferentes dimensiones de la satisfacción laboral y la cualificación del trabajo (relacionado o no con 
los currículos académicos previos). Se ha realizado utilizando Cuestionario de Satisfacción Laboral S20/23. Los resultados han sido significativos, confirmándose la hipótesis: hemos encontrado mayor satisfacción laboral en los sujetos que desarrollan trabajos cualificados y menor satisfacción en los que desarrollan trabajos no cualificados. Palabras clave: Cualificación; Satisfacción laboral; Formación previa.

Efectos de la turnicidad sobre la ansiedad y la salud psicológica en profesionales sanitarios de centros hospitalarios (Lizarte, Montero, Peral y Postigo, 2012). Resumen: El objetivo de este estudio correlacional es analizar el efecto que tiene el turno rotatorio sobre la ansiedad y la salud psicológica autopercibida del personal sanitario de enfermería, frente a aquellos profesionales de la misma categoría que tienen un turno fijo. Para ello se administraron dos cuestionarios que recogían las variables de interés, a una muestra de 140 profesionales de enfermería que trabajan en los hospitales de la provincia de Granada. Los resultados han mostrado que no hay evidencia significativa entre la ansiedad y la salud psicológica autopercibida sobre la turnicidad, sin diferencias relevantes con el turno fijo. Se concluye que hay una tendencia a padecer más trastornos de ansiedad entre aquellos profesionales sanitarios que llevan más años en la profesión. Palabras clave: Turnicidad; Ansiedad; Salud percibida; Enfermería.

\begin{abstract}
¿Depende la satisfacción estudiantil de la calidad real de la facultad o de la percepción individual de esta? Análisis comparativo entre facultades privadas y públicas (Pérez, López, Couto y Navarro, 2012). Resumen: El objetivo de este estudio es conocer la satisfacción estudiantil en relación a las universidades públicas y privadas. Es un estudio correlacional, realizado en la Universidad de Granada en la escuela universitaria de magisterio La Inmaculada y en la de facultad de ciencias de la educación. Participaron 111 alumnos entre ambas facultades que completaron un cuestionario sobre satisfacción estudiantil. Los principales resultados exponen que a pesar de no obtener diferencias significativas en la satisfacción global, sí lo hacen en distintos aspectos, obteniendo mayor satisfacción los estudiantes de la Facultad privada, en competencias profesionales y servicio administrativo, en comparación con los de la pública que obtienen mayores puntuaciones en satisfacción con el ambiente en la facultad y la biblioteca. Palabras Clave: Satisfacción estudiantil; Universidad Pública; Universidad Privada.
\end{abstract}

Las habitaciones de la Dislalia (Moreno y Ramírez, 2012). Resumen: La primera parte de este artículo la hemos dedicado a exponer algunos aspectos que consideramos básicos para conocer en qué consiste la dislalia. Hemos desarrollado con más profundidad la dislalia funcional porque es uno de los trastornos de habla que actualmente está aumentando su porcentaje de aparición en los colegios. El resto del artículo está dedicado a concienciar sobre la importancia que tiene el entorno familiar en el desarrollo lingüístico del/a niño/a y a ofrecer algunas orientaciones y ejercicios que permita a la familia estimular este desarrollo utilizando el tiempo que emplean para realizar actividades de su vida cotidiana. Palabras clave: Desarrollo del lenguaje; Trastorno del habla; Familia; Dislalia.

Guía de intervención para un alumno con Hipoacusia Neurosensorial (Segura Azor (Universidad de Granada). Resumen: Este estudio de caso se centra en un niño con hipoacusia neurosensorial. Se detalla cómo se detectó y qué pasos se siguieron en su tratamiento. En el equipo reintervención, contamos con una maestra de Pedagogía Terapéutica (PT) y dos profesionales de Audición y Lenguaje ( $A L)$. El sujeto ha tenido una evolución muy adecuada, a partir de los objetivos y actividades propuestas por el equipo a lo largo de su etapa escolar, las cuales se describen en el presente trabajo. Este estudio de caso pretende servir de guía a futuros maestros y maestras que trabajen ante una problemática similar. Palabras clave: Estudio de caso; Sordera; Educación de sordos; Lenguaje de signos.

El síndrome de Dandy Walker y su intervención en la infancia (García, 2012). Resumen: El Síndrome de Dandy Walker es una anomalía congénita del cerebelo y del IV ventrículo, que aparece normalmente en la infancia y recibe múltiples nominaciones. 
Su característica más común es la hidrocefalia y en la mayoría de los casos, las causas se desconocen. El artículo se centra en gran parte en el análisis de un caso práctico de un niño de cinco años, con dificultades en la movilidad debido al retraso psicomotor y sin habla, cuyo tratamiento está basado en el control de los movimientos, en la estimulación del balbuceo y en el aprendizaje de un sistema alternativo de comunicación. Palabras clave: Síndrome de Dandy Walker; Infancia; Lengua; Necesidades educacionales; Técnicas de comunicación.

Trabajando de forma cooperativa en la escuela: abriendo la puerta a las familias (García, 2012). Resumen: Este estudio pretende mostrar la importancia del trabajo cooperativo en la escuela, en especial del relacionado con la participación de los padres y la familia de los alumnos con el profesorado y el centro educativo. Por otro lado, se propondrán variadas acciones y actividades educativas prácticas que se pueden llevar a cabo en el mismo para conseguir la relación cooperativa familia-escuela y alcanzar así el clima óptimo para el desarrollo integral del alumno y una educación de calidad que serán los principales objetivos perseguidos. Todo ello se hará en el marco del respeto y la integración de todos los sujetos implicados. Palabras clave: Relación padres-escuela; Familia; Participación de los padres; Enseñanza en equipo; Cooperación educacional.

El uso de técnicas de animación sociocultural en la Universidad mejora las relaciones interpersonales entre profesores y estudiantes en la universidad (López, Platero, Aguilar y Rubia, 2012). Resumen: La relación existente entre el profesor y los alumnos puede depender de muchas circunstancias. De entre ellas, este estudio se centra en confirmar que el uso de técnicas de animación sociocultural en el aula hace que se vean mejoradas las relaciones interpersonales entre estos agentes educativos. El procedimiento metodológico seguido se centra en un enfoque interpretativo-descriptivo mediante el uso de unos cuestionarios como técnicas de recogida de datos. Los resultados encontrados confirman nuestra hipótesis inicial y a la vez nos abren las puertas hacia futuras líneas de investigación. Palabras clave: Animación sociocultural; Relaciones interpersonales; Innovación educativa; Comunicación interpersonal; Grupos.

La educación diferenciada en Primaria, ¿se puede llamar coeducación? (Sánchez, 2012). Resumen: En este trabajo se tiene como referencia el aprendizaje diferenciado en la etapa de Primaria, y tras tener en cuenta diversas teorías tanto a favor como en contra de la misma, podemos realizar un análisis exhaustivo de si realmente dicho aprendizaje logra la coeducación o no, en el aula. Como conclusión podemos sostener que existen pocos estudios que abalen dicha manera de entender la educación, además como hemos podido observar se tiende a generalizar en la enseñanza de niñas y de niños como un todo, sin tener en cuenta las características esenciales de cada persona. Palabras clave: Educación; Diferenciación; Sexos; Métodos; Posibilidades.

Consumo de sustancias adictivas y efectos en las relaciones intergrupales y personales en estudiantes universitarios (Pastrana, Manrubia y Muñoz, 2012). Resumen: Tras una primera revisión bibliográfica se llega a la conclusión de que, en el ámbito del estudiante universitario español, no se han realizado estudios acerca de las consecuencias del consumo de sustancias adictivas en relación a las relaciones interpersonales. Nuestro objetivo es analizar los efectos del consumo de sustancias adictivas dentro de las relaciones intergrupales en el ámbito universitario para que, posteriormente, esta investigación pueda ser llevada a cabo en otros ámbitos. La muestra empleada, que ha sido seleccionada de manera aleatoria, queda organizada en 50 estudiantes, a los cuales se les ha pasado un cuestionario relacionado con el tema investigado. Nuestros resultados apoyan el efecto que causa el consumo de sustancias adictivas, a través de las diferentes variables estudiadas, en la población universitaria. Palabras claves: Sustancias adictivas; Consumo; Consecuencia; Relaciones intergrupales de género; Concienciación.

Las redes sociales influyen en el comportamiento de los universitarios (Jabalera, Morey, Rodríguez y Sánchez, 2012). Resumen: Las redes sociales son un medio muy utilizado hoy en día por el colectivo universitario, de este modo nos centramos en 
investigar su influencia en el comportamiento de éstos, para comprobar hasta qué punto se dejan de lado las relaciones interpersonales por la utilización de éstas, así como las actividades más utilizadas en las mismas, y las que se dejan de realizar por su utilización. Nos centramos en un colectivo de la universidad de Granada. Palabras clave: Redes sociales; Relaciones interpersonales; Comunicación; Influencia social; Disonancia cognitiva.

Las mujeres viven la relación romántica diferente al hombre (García, Garnica, González, Márquez, Martín, Pérez y Vico, 2012). Resumen: Desde hace unas décadas, han emergido una serie de teorías que estudian las relaciones románticas y los estilos de amor. En base a estas teorías, nos ha parecido interesante estudiar las relaciones románticas de 120 estudiantes de la Facultad de Ciencias de la Educación de la Universidad de Granada y confirmar si existe relación con la diferencia de género, comprobando si difieren en la importancia que otorgan a los diferentes estilos de amor. Para ello, se administró la Escala de Actitudes sobre el Amor (LAS). Los resultados indican que entre las mujeres el estilo de amor más aceptado es Eros mientras que en los hombres es Ludus. En los estilos Ágape y Manía se muestra una clara diferencia entre mujeres y hombres, ya que estos obtienen mayor puntuación de la esperada. Se muestra una indiferencia, sin distinción de género, en los estilos de amor Pragma y Storge. Se analizan y discuten los resultados. Palabras claves: Relación romántica; Estilos de amor; Género.

La medida de la empatia en el alumnado de la facultad de ciencias de la educación de Granada (Artacho, López, Molina, Ortiz, Rosado, Ruiz y Sillero, 2012). Resumen: El objetivo principal de este trabajo de investigación ha sido analizar la capacidad empática de 100 universitarios de la Facultad de Ciencias de la Educación de Granada. Para ello hemos utilizado el "Interpersonal Reactivity Index" (IRI), que es uno de los cuestionarios más utilizados para evaluar esta capacidad. Este instrumento incluye dos subescalas dedicadas a factores cognitivos, la toma de perspectiva (PT) y la fantasía (FS) y otras dos a factores emocionales, la preocupación empática (EC) y el malestar personal (PD). Palabras Clave: Empatía; Toma de perspectiva; Fantasía; Preocupación empática; Malestar personal.

Las tic en la educación superior como vía de formación y desarrollo competencial en la sociedad del conocimiento (Molina, 2012). Resumen: La sociedad del conocimiento, nos pone a prueba en relación a las destrezas, conocimientos y habilidades que adquirimos. El ámbito educativo ha de avanzar al compás de esta sociedad desde la primera de las etapas hasta el final de ellas, valiéndose de las TIC. A través del presente trabajo se pretende analizar la interacción comunicativa establecida entre docentes y discentes a través de los espacios virtuales en la Educación Superior, profundizando en sus posibilidades y destacando la necesidad de flexibilizar la educación. A la vez, se muestran una serie de experiencias renovadoras y unas vías de continuidad en el futuro. Palabras clave: Tutor virtual; Espacio virtual; Aprendizaje virtual; TIC; Educación Superior.

Percepción de la inclusión educativa en el contexto universitario: un estudio aproximativo en la Facultad de Ciencias de la Educación de la Universidad de Granada (Conde, 2012). Resumen: El presente artículo pretende realizar un análisis aproximativo respecto al grado de aplicación de la inclusión educativa en la Facultad de Ciencias de la Educación de la ciudad de Granada. ¿Es consciente el alumnado y el profesorado de esta corriente que se está desarrollando desde hace tiempo en el sistema educativo de otros países y también en el nuestro; En qué grado conciben la comunidad universitaria que está llevándose a cabo? Primera parte, de un estudio aproximativo centrado en la percepción del alumnado, que posteriormente se complementará con la percepción del profesorado. Palabras clave: Educación universal; Acceso a la educación; Inclusión educativa; Enseñanza Superior.

Calidad educativa y espacios de trabajo universitarios (Fernández, Gámiz, García, Moraga, Peña y Porras, 2012). Resumen: La sociedad actual avanza a pasos 
agigantados, circunstancia que exige de nuestro modelo educativo un cambio paralelo a dichos avances. La educación es uno de los motores de cambio y como tal debe dar respuesta a las exigencias que se le planteen. Es mucha la bibliografía que podemos encontrar acerca de la calidad educativa, pero muy poca de ésta se centra en la evaluación de la calidad de los espacios educativos como tales. Desde este estudio se pretende analizar los puntos de vista y exigencias de los estudiantes de la Facultad de Ciencias de la Educación de la Universidad de Granada respecto a dichos espacios educativos. Palabras clave: Universidad; Calidad educativa; Espacios educativos; Ambiente educativo.

EI Mindfulness como método para la mejora de las relaciones interpersonales (Rodríguez de Medina, 2012). Resumen: Muchos de los problemas sociales se remontan a las dificultades de comunicación interpersonal. Este ensayo examina el potencial de la relación entre los estados de la mente, su anatomía y las interacciones sociales seguido por la eficacia de los programas Mindfulness para la mejora de los males sociales. Palabras claves: Relaciones interpersonales; Mindfulness; Emoción; Neurobiología; Apego.

La interacción comunicativa en el proceso de enseñanza-aprendizaje (Márquez, 2012). Resumen: Tomando como referencia el proceso de interacción comunicativa, que alude al sistema de transmisión de mensajes o información entre personas, y teniendo en cuenta los tipos de lenguajes implicados en el proceso comunicativo, en este trabajo se abordarán las diferentes formas de interacción comunicativa -formal y espontáneoque pueden llegar a darse en el aula entre profesorado y alumnado, tanto de forma interpersonal como grupal, así como sus implicaciones didácticas. De igual modo, se realizará un breve recorrido sobre los estilos de profesorado y por consiguiente, los estilos de comunicación didáctica que favorecen un tipo u otro de interacción entre el profesorado y el alumnado. Palabras clave: Comunicación; Interacción; Enseñanza; Aprendizaje.

Universidad y empredimiento: Un caso de estudio en la Facultad de Ciencias Económicas y Empresariales de la Universidad de Granada (Ruiz, Cabeza y Briano, 2012). Resumen: Con el objetivo de fomentar una cultura emprendedora dentro del ámbito educativo, se desarrollaron prácticas en las cuales los estudiantes de la licenciatura en administración y dirección de empresas, debían realizar las distintas etapas del plan de empresa que el emprendedor tiene en cuenta en el momento de poner en marcha su negocio. En este trabajo, analizamos esta experiencia en emprendimiento, destacando el importante desafío que tienen las instituciones educativas en desarrollar programas, en los cuales, los estudiantes puedan contar con herramientas que les sirvan a la hora de emprender su propio negocio en un mercado laboral competitivo. Con el fin de contribuir al desarrollo económico y social del país. Observamos que antes de esta practica, la mayoría de los alumnos no habían tenido una experiencia de emprendimiento es su carrera universitaria. Además, al finalizar dicha práctica más de la mitad de los estudiantes desean continuar con su proyecto de empresa. Palabras clave: Emprendimiento; Mercado laboral; Universidad.

La competencia comunicativa en personas que presentan parálisis cerebral (Rosado, 2012). Resumen: A pesar de los numerosos intentos por definir universalmente la Parálisis Cerebral, todavía no se ha conseguido una definición que una todas las opiniones acerca de ésta, aunque sí existen puntos en común en la mayoría de ellas. Todas las personas, para poder desenvolverse en su contexto social, deben desarrollar su capacidad de comunicación, especialmente las personas con parálisis cerebral. Esta capacidad se debe potenciar, desde el desarrollo de la lengua por parte de los centros educativos, a través del desarrollo de la competencia comunicativa ya sea a través del lenguaje oral o a través de los sistemas alternativos/aumentativos de comunicación. Palabras clave: discapacidad, persona con discapacidad, comunicación, comunicación alternativa, competencia comunicativa. 


\section{Qué supone el proyecto para la mejora del aprendizaje}

Este proyecto supone una mejora global en el proceso de enseñanza-aprendizaje, porque implica de una forma participativa y creativa a ambas partes del proceso educativo, docentes y estudiantes. El profesorado orienta y guía al alumnado durante el proceso de aprendizaje de los contenidos necesarios para que adquiera las competencias necesarias de la propia área del conocimiento; pero el profesorado, también, es responsable garantizar la mayor calidad en los trabajos académicos. La publicación de un trabajo académico es un reconocimiento público a los autores; pero este proyecto también conlleva una mejora en eficiencia y satisfacción, porque el estudiante puede sentir que su rendimiento es útil. También forma parte de un proceso de enseñanza-aprendizaje sociocultural consciente de la importancia de la difusión del conocimiento.

\section{Conclusiones}

Los resultados obtenidos justifican la necesidad de potenciar la comunicación entre docentes y estudiantes para construir el conocimiento de manera social o colaborativa. Esto se puede implementar desde la simpatía como proceso de escucha que supone ponerse en el lugar del otro y considerarlo un igual aunque los roles de docente y estudiante sean diferentes. Por lo tanto, en la Educación Superior reconocer los trabajos que realizan los/as estudiantes está significativamente relacionado con la motivación y el pensamiento creativo. (García-Ramírez, 2012)

La divulgación del conocimiento adquirido responde a la demanda social; además de ser un vehículo de reconocimiento y eficiencia de una Educación de Excelencia Visible. Por lo tanto, se plantea la creación de ReiDoCrea, Revista electrónica de investigación y Docencia Creativa, partiendo de los recursos existentes. (Fiorini, García Ramírez, 2013)

Para finalizar todas las publicaciones en la revista están sujetas a la licencia Creative Commons Attribution 4.0 International, se adhiere a los principios y procedimientos dictados por el Committee on Publication Ethics "COPE", deposita todos los artículos en el Repositorio de la Universidad de Granada "Digibug" y ha sido indexada en el catálogo de Latindex y en el Google Académico.

\section{Referencias}

Artacho Castilla, F.; et al. (2012). La medida de la empatía en el alumnado de la Facultad de Ciencias de la Educación de Granada. ReiDoCrea, 1, 101-105. http://hdl.handle.net/10481/21968

Asenjo Fenoy, A.; Banqueri López, M.; De Dios Chacón, M. (2012). Cualificación y satisfacción laboral: un estudio sobre los empleos para los que hemos sido formado previamente. ReiDoCrea, 1, 4-11. http://hdl.handle.net/10481/21938 
Conde Lacárcel, A. (2012). Percepción de la inclusión educativa en el contexto universitario: un estudio aproximativo en la FCEE de Granada. ReiDoCrea, 1, 115-125. http://hdl.handle.net/10481/20634

Fernández Pérez, R.; et al. (2012). Calidad educativa y espacios de trabajo universitarios. ReiDoCrea, 1, 126-131. http://hdl.handle.net/10481/21986

Fiorini, M.; Garcia-Ramirez, JM. (2013). Cap. 5: Técnicas de grupo y creatividad aplicadas en el ámbito universitario,117-147. En Villena Martinez, MD.; Muñoz Garcia, A. (2013). Recursos para la tutoría en el aula universitaria. Granada: Editorial Universidad de Granada.

Gallardo Pastrana, M.E.; Manrubia Prados, C.M.; Muñoz Palomino, R. (2012). Consumo de sustancias adictivas y efectos en las relaciones intergrupales y personales en estudiantes universitarios. ReiDoCrea, 1, 80-87. http://hdl.handle.net/10481/21975

García Caballero, I.M. (2012). El síndrome de Dandy Walker y su intervención en la infancia. ReiDoCrea, 1, 52-58. http://hdl.handle.net/10481/21943

García Carmona, M. (2010). Trabajando de forma cooperativa en la escuela: abriendo la puerta a las familias. ReiDoCrea, 1, 59-66. http://hdl.handle.net/10481/21944

García Palma, M.E.; et al. (2012). Las mujeres viven la relación romántica diferente al hombre. ReiDoCrea, 1, 95-100. http://hdl.handle.net/10481/21967

Garcia-Ramirez, J.M. (2011). Una reconsideración de la excelencia visible en la educación superior: la escucha empática. Andaluciaeduca, 66, 84. http://www.andaluciaeduca.com/hemeroteca/ae digital66.pdf

García-Ramírez, J.M. (2012). Docencia-Creativa: una estrategia de motivación a través de la difusión del conocimiento. Reidocrea, 1, 1-3. http://hdl.handle.net/10481/33571

García-Ramírez, J.M. (2012). La comunicación, clave de excelencia visible en la Educación Superior. Journal for Educators, Teachers and Trainers, 3, 25-36. http://hdl.handle.net/10481/22300

Garcia-Ramirez, J.M. (2012). Las Tecnologías de la Información y la Comunicación, TIC, en la educación universitaria. Andaluciaeduca, 76, 77. http://www.andaluciaeduca.com/hemeroteca/ae_digital76.pdf

García-Ramírez, J.M. (ed.). (2012). ReiDoCrea: volumen 1. Granada: Universidad de Granada. http://hdl.handle.net/10481/33702

García-Ramírez, J.M.; García-Sempere, P.J.; Fiorini, M. (coords.). (2012). Docencia universitaria y creatividad. Granada: Universidad de Granada. http://hdl.handle.net/10481/33193

Garcia-Ramirez, J.M.; Perez-Villen, MP. (2003). Educación y convivencia, 555-557. En Alvaraz-Rodriguez, J.; Casares-Garcia, P.; Luengo-Navas, J. (2003). Participación, Convivencia y Ciudadanía. Granada: Ediciones Osuna. ISBN 13: 978-84-95805-18-8 
Herrero Márquez, P. (2012). La interacción comunicativa en el proceso de enseñanzaaprendizaje. ReiDoCrea, 1, 138-143. http://hdl.handle.net/10481/21983

Jabalera Sierra, P.M.; Morey Amer, M.M.; Rodríguez Bonachera, A.; Sánchez Molina, A.B. (2012). Las redes sociales influyen en el comportamiento de los universitarios. ReiDoCrea, 1, 88-94. http://hdl.handle.net/10481/21966

Lizarte Castillo, M.; Montero Madej, A.; Peral Bueno, N.; Postigo Higueras, E. (2012). Efectos de la turnicidad sobre la ansiedad y salud psicológica en profesionales sanitarios de centros hospitalarios. ReiDoCrea, 1, 21- 28. http://hdl.handle.net/10481/21939

López Serrano, C.J.; et al. (2012). El uso de técnicas de animación sociocultural en la Universidad mejora las relaciones interpersonales entre profesores y estudiantes en la Universidad. ReiDoCrea, 1, 67-75. http://hdl.handle.net/10481/21945

Molina Ramírez, A.M. (2012). Las TIC en la educación superior como vía de formación y desarrollo competencial en la sociedad del conocimiento. ReiDoCrea, 1, 106114. http://hdl.handle.net/10481/21977

Moreno González, R.; Ramírez Villegas, M.A. (2012). Las habitaciones de la dislalia. ReiDoCrea, 1, 38-45. http://hdl.handle.net/10481/21941

Pérez Zamora, L.A.; López López, M.C.; Couto Olivares, J.M.; Navarro Luque, G. (2012). ¿Depende la satisfacción estudiantil de la calidad real de la facultad o de la percepción individual de ésta? Análisis comparativo entre facultades privadas y públicas. ReiDoCrea, 1, 29-37. http://hdl.handle.net/10481/21940

Rodríguez de Medina Quevedo, I. (2012). El Mindfulness como método para la mejora de las relaciones interpersonales. ReiDoCrea, 1, 132-137. http://hdl.handle.net/10481/21978

Rosada Ayala, J.I. (2012). La competencia comunicativa en personas que presentan parálisis cerebral. ReiDoCrea, 1, 158-163. http://hdl.handle.net/10481/21996

Ruiz Jiménez, J.M.; Cabeza Pullés, D.; Briano Turrent, G.C. (2012). Universidad y empredimiento: un caso de estudio en la Facultad de Ciencias Económicas y Empresariales de la UGR. ReiDoCrea, 1, 144-157. http://hdl.handle.net/10481/21988

Sagripanti Mazuquin, O.G.; et al. (2012). El efecto del burnout en la antigüedad en el puesto de trabajo: análisis mediacional de la ansiedad. ReiDoCrea, 1, 4-11. http://hdl.handle.net/10481/21909

Sánchez Arco, D.V. (2012). La educación diferenciada en Primaria, ¿se puede llamar coeducación? ReiDoCrea, 1, 76-79. http://hdl.handle.net/10481/21965

Segura Azor, E.A. (2012). Guía de intervención para un alumno con hipoacusia neurosensorial. ReiDoCrea, 1, 46-51. http://hdl.handle.net/10481/21942 